\title{
Modeling and Motion Planning for Mechanisms on a Non-Inertial Base
}

\author{
Pål J. From ${ }^{1} \quad$ Vincent Duindam $^{2} \quad$ Jan T. Gravdahl ${ }^{1} \quad$ Shankar Sastry $^{2}$ \\ ${ }^{1}$ Department of Eng. Cybernetics, Norwegian Univ. of Science and Technology, Trondheim, Norway \\ ${ }^{2}$ Department of EECS, University of California, 253 Cory Hall, Berkeley, CA 94720-1770, USA \\ \{from,tommy.gravdahl\}@itk.ntnu.no v.duindam@ieee.org sastry@eecs.berkeley.edu
}

\begin{abstract}
Robotic manipulators on ships and platforms suffer from large inertial forces due to the non-inertial motion of the ship or platform. When operating in high sea state, operation of such manipulators can be made more efficient and robust if these non-inertial effects are taken into account in the motion planning and control systems.

Motivated by this application, we present a rigorous and singularity-free formulation of the dynamics of a robotic manipulator mounted on a non-inertial base. We extend the classical dynamics equations for a serial manipulator to include the 6-DoF motion of the non-inertial base. Then, we show two examples of a 1-DoF and a 4-DoF manipulator to illustrate how these non-inertial effects can be taken into account in the motion planning.
\end{abstract}

\section{INTRODUCTION}

The use of unmanned and autonomous vehicles operating in hostile environments has shown both to be cost efficient and to protect humans from potentially dangerous situations. One such hostile environment is high sea state. We look into the case when a manipulator mounted on a ship or a platform is required to operate independently of the sea state. Large inertial forces may influence the manipulator and make the operation inaccurate, extremely energy demanding, or impossible due to torque limits. The inertial forces thus need to be taken into account in both the path planning and control of the robot.

Ships and oil platforms are expected to become increasingly unmanned in the future and hence the need for continuously operating robots for surveillance, maintenance, and operation will grow [1], [2]. All these tasks become increasingly important in harsh environments such as high sea state. To be able to continue operation under these conditions, a good understanding is needed of the effects of the inertial forces due to the motion of the ship or platform. We therefore develop the dynamic equations of the robotic manipulator including these effects.

Research on several related topics can be found in literature. Love et al. [1] addressed the impact of wave generated disturbances on the tracking control of a manipulator mounted on a ship based on the classical Lagrangian approach. Repetitive learning control was used and performance was improved for purely periodic motions, but no formal derivation of the dynamics equations was presented. The use of cable robots for loading and unloading cargo between two ships has also been addressed by Kitarovic et al. [2] and Oh et al. [3]. In the Ampelmann project [4], a Stewart platform is mounted on a ship and is used to compensate

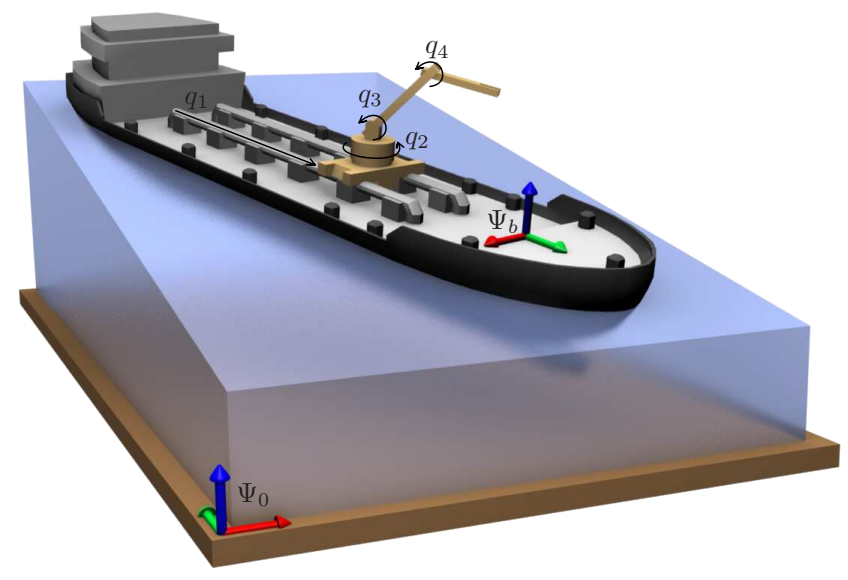

Fig. 1. Model setup for a four-link robot attached to a non-inertial base with coordinate frame $\Psi_{b}$. Frame $\Psi_{0}$ denotes the inertial reference frame.

for the motion of the ship by keeping the platform still with respect to the world frame. Lebans et al. [5] give a cursory description of a telerobotic shipboard handling system, and Kosuge et al. [6], [7] addresses the control of robots floating on the water utilizing vehicle restoring forces. Other related research areas are macro/micro manipulators [8], [9], underwater vehicle/manipulator systems [10] and spacecraft/manipulator systems [11]. Most previous work deals with robots mounted on a free-floating base. There is, however, an important difference between modeling a robot on a forced and a free-floating base. A forced base motion will add inertial forces to the dynamic equations that do not arise in free-floating case, such as spacecraft/manipulator systems and manipulators on small AUVs.

Our approach differs from previous work in that the dynamic equations are derived for rigid multibody systems including both Euclidean joints and generalized joints with configuration spaces different from $\mathbb{R}^{n}$. We follow the generalized Lagrangian approach presented in Duindam et al. [12], [13], which allows us to combine the Euclidean joints (the manipulator) and more general joints (the base), i.e. joints that can be described by the Lie group $S E(3)$ or one of its ten subgroups. In our case, the transformation from the inertial frame to the base frame (the platform) is represented as a "free motion" joint described by the Lie group $S E(3)$. We also show through the examples how the base motion can be expressed as subgroups of $S E(3)$, in our case $S O(2)$.

For marine vessels in high sea state, very large inertial 
forces are added to the manipulator dynamics. To illustrate the effect of the inertial forces and how these appear in the robot equations, we look into the problem of finding the optimal trajectory in terms of actuator torque. There are many motivations for doing this. First of all the wear and tear on the manipulator is reduced and, for cooperative manipulation, the possibility of breaking an object manipulated by two robots is also reduced. Secondly, the solution is more energy efficient as the inertial forces will, if possible, contribute to the desired motion instead of working against it. The final motivation is that a good understanding of the effects of the inertial forces on the dynamic equations is essential in tasks that require high accuracy and the need to compensate for these effects [1].

We assume that the motion of the free moving base is forced externally by forces unknown to us and that the pose, velocity, and acceleration of the base relative to the inertial world are known for all times. This means we also assume that the motion of the robot does not influence the motion of the base. The motion of the base will add inertial forces to the dynamics equations of the robot and the pose of the base will influence the gravitational forces acting on each link. Finally, we assume the robot to be an ideal rigid frictionless mechanism with purely torque driven actuators.

Given these assumptions, we consider the following two problems: first, we derive the dynamic equations describing the motion of the robot under the influence of the non-inertial base motion. Second, we consider the path planning problem of finding the trajectory between two given configurations for a given base motion. Intuitively, the optimal solution is the trajectory for which the inertial forces help accelerate and decelerate the robot as much as possible, such that little control torque is required.

\section{Multibody DYNAMics With A NON-INERTIAL BASE}

We extend the classical dynamics equations for a serial manipulator arm with 1-DoF joints to include the forced 6DoF motion of the non-inertial base.

\section{A. Manipulator kinematics on a non-inertial base}

Consider the setup of Figure 1 describing a general $n$-link robot manipulator arm attached to a moving base. Choose an inertial coordinate frame $\Psi_{0}$, a frame $\Psi_{b}$ rigidly attached to the moving base, and $n$ frames $\Psi_{i}$ (not shown) attached to each link $i$ at the center of mass with axes aligned with the principal directions of inertia. Finally, choose a vector $q \in \mathbb{R}^{n}$ that describes the configuration of the $n$ joints.

Using standard notation [14], we can describe the pose of each frame $\Psi_{i}$ relative to $\Psi_{0}$ as a homogeneous transformation matrix $g_{0 i} \in S E(3)$ of the form

$$
g_{0 i}=\left[\begin{array}{cc}
R_{0 i} & p_{0 i} \\
0 & 1
\end{array}\right] \in \mathbb{R}^{4 \times 4}
$$

with rotation matrix $R_{0 i} \in S O(3)$ and translation vector $p_{0 i} \in \mathbb{R}^{3}$. This pose can also be described using the vector of joint coordinates $q$ as

$$
g_{0 i}=g_{0 b} g_{b i}=g_{0 b} g_{b i}(q)
$$

The base pose $g_{0 b}$ and the joint positions $q$ thus fully determine the configuration state of the robot.

In a similar way, the spatial velocity of each link can be expressed using twists [14]:

$$
V_{0 i}^{0}=\left[\begin{array}{c}
v_{0 i}^{0} \\
\omega_{0 i}^{0}
\end{array}\right]=V_{0 b}^{0}+V_{b i}^{0}=\operatorname{Ad}_{g_{0 b}}\left(V_{0 b}^{b}+J_{i}(q) \dot{q}\right)
$$

where $v_{0 i}^{0}$ and $\omega_{0 i}^{0}$ are the linear and angular velocities, respectively, of link $i$ relative to the inertial frame, $J_{i}(q) \in$ $\mathbb{R}^{6 \times n}$ is the geometric Jacobian of link $i$ relative to $\Psi_{b}$, the adjoint is defined as $\operatorname{Ad}_{g}:=\left[\begin{array}{cc}R & \hat{p} R \\ 0 & R\end{array}\right] \in \mathbb{R}^{6 \times 6}$, and $\hat{p} \in \mathbb{R}^{3 \times 3}$ is the skew symmetric matrix such that $\hat{p} x=p \times x$ for all $p, x \in \mathbb{R}^{3}$. The velocity state is thus fully determined given the twist $V_{0 b}^{b}$ of the base and the joint velocities $\dot{q}$.

\section{B. Manipulator dynamics on a non-inertial base}

The previous section shows how the kinematics of the system can be naturally described in terms of the (global) state variables $g_{0 b}, q, V_{0 b}^{b}$, and $\dot{q}$. We now derive the dynamics equations for the system in terms of these state variables. We first assume the base to be free-moving under the influence of some prescribed external wrench $F$, and then restrict the base motion to be kinematically constrained.

To derive the dynamics of the complete mechanism (including the 6-DoF between $\Psi_{0}$ and $\Psi_{b}$ ), we follow the generalized Lagrangian method introduced by Duindam et al. [12], [13]. This method gives the dynamics equations for a general mechanism described by a set $Q=\left\{Q_{i}\right\}$ of configuration states $Q_{i}$ (not necessarily Euclidean), a vector $v$ of velocity states $v_{i} \in \mathbb{R}^{n_{i}}$, and several mappings that describe the local Euclidean structure of the configuration states and their relation to the velocity states. More precisely, the neighborhood of every state $\bar{Q}_{i}$ is locally described by a set of Euclidean coordinates $\phi_{i} \in \mathbb{R}^{n_{i}}$ as $Q_{i}=Q_{i}\left(\bar{Q}_{i}, \phi_{i}\right)$ with $Q_{i}\left(\bar{Q}_{i}, 0\right)=\bar{Q}_{i}$, and there exist differentiable matrices $S_{i}$ such that we can write $v_{i}=S_{i}\left(Q_{i}, \phi_{i}\right) \dot{\phi}_{i}$ for every $Q_{i}$.

Given a mechanism with coordinates formulated in this generalized form, we can write its kinetic energy as $U_{k}(Q, v)=\frac{1}{2} v^{T} M(Q) v$ with $M(Q)$ the inertia matrix in coordinates $Q$. The dynamics of this system then satisfy

$$
M(Q) \dot{v}+\bar{C}(Q, v) v=\bar{\tau}
$$

with $\tau$ the vector of gravitational, friction, and other external torques (collocated with $v$ ), and $\bar{C}(Q, v)$ the matrix describing Coriolis and centrifugal forces and given by

$$
\begin{gathered}
\bar{C}_{i j}(Q, v):=\left.\sum_{k, l}\left(\frac{\partial M_{i j}}{\partial \phi_{k}} S_{k l}^{-1}-\frac{1}{2} S_{k i}^{-1} \frac{\partial M_{j l}}{\partial \phi_{k}}\right)\right|_{\phi=0} v_{l} \\
\quad+\left.\sum_{k, l, m, s}\left(S_{m i}^{-1}\left(\frac{\partial S_{m j}}{\partial \phi_{s}}-\frac{\partial S_{m s}}{\partial \phi_{j}}\right) S_{s k}^{-1} M_{k l}\right)\right|_{\phi=0} v_{l}
\end{gathered}
$$

More details and proofs can be found in references [12] and [13].

To apply this general result to systems of the form of Figure 1, we write $Q=\left\{g_{0 b}, q\right\}$ as the set of configuration states, and $v=\left[\begin{array}{c}V_{0 b}^{b} \\ \dot{q}\end{array}\right]$ as the vector of velocity states. 
The local Euclidean structure for the state $g_{0 b}$ is given by exponential coordinates [14], while the state $q$ is globally Euclidean of itself. Mathematically, we can express configurations $\left(g_{0 b}, q\right)$ around a fixed state $\left(\bar{g}_{0 b}, \bar{q}\right)$ as

$$
\begin{gathered}
g_{0 b}=\bar{g}_{0 b} \exp \left(\sum_{j=1}^{6} b_{j}\left(\phi_{b}\right)_{j}\right) \\
q_{i}=\bar{q}_{i}+\phi_{i} \quad \forall i \in\{1, \ldots, n\}
\end{gathered}
$$

with $b_{j}$ the standard basis elements of the Lie algebra $s e(3)$. The corresponding matrices $S_{i}$ can be collected in one blockdiagonal matrix $S$ given by [15]

$S(Q, \phi)=\left[\begin{array}{cc}\left(I-\frac{1}{2} \operatorname{ad}_{\phi_{b}}+\frac{1}{6} \operatorname{ad}_{\phi_{b}}^{2}-\ldots\right) & 0 \\ 0 & I\end{array}\right] \in \mathbb{R}^{(6+n) \times(6+n)}$

with $\operatorname{ad}_{p}=\left[\begin{array}{cc}\hat{p}_{4} \ldots 6 & \hat{p}_{1 \ldots 3} \\ 0 & \hat{p}_{4} \ldots 6\end{array}\right] \in \mathbb{R}^{6 \times 6}$ for $p \in \mathbb{R}^{6}$. This shows that the choice of coordinates $(Q, v)$ has the required form.

From expression (3) for the twist of each link in the mechanism, we can derive an expression for the total kinetic energy. Let $I_{i} \in \mathbb{R}^{6 \times 6}$ denote the constant positive-definite diagonal inertia tensor of link $i$ expressed in $\Psi_{i}$. The kinetic energy $U_{k, i}$ of link $i$ then follows as

$$
\begin{aligned}
U_{k, i} & =\frac{1}{2}\left(V_{0 i}^{i}\right)^{T} I_{i} V_{0 i}^{i} \\
& =\frac{1}{2}\left(V_{0 b}^{b}+J_{i}(q) \dot{q}\right)^{T} \operatorname{Ad}_{g_{i b}}^{T} I_{i} \operatorname{Ad}_{g_{i b}}\left(V_{0 b}^{b}+J_{i}(q) \dot{q}\right) \\
& =\frac{1}{2}\left[\left(V_{0 b}^{b}\right)^{T} \dot{q}^{T}\right] M_{i}(q)\left[\begin{array}{c}
V_{0 b}^{b} \\
\dot{q}
\end{array}\right]=\frac{1}{2} v^{T} M_{i}(q) v
\end{aligned}
$$

with

$$
M_{i}(q):=\left[\begin{array}{cc}
\operatorname{Ad}_{g_{i b}}^{T} I_{i} \operatorname{Ad}_{g_{i b}} & \operatorname{Ad}_{g_{i b}}^{T} I_{i} \operatorname{Ad}_{g_{i b}} J_{i} \\
J_{i}^{T} \operatorname{Ad}_{g_{i b}}^{T} I_{i} \operatorname{Ad}_{g_{i b}} & J_{i}^{T} \operatorname{Ad}_{g_{i b}}^{T} I_{i} \operatorname{Ad}_{g_{i b}} J_{i}
\end{array}\right]
$$

The total kinetic energy of the mechanism is given by the sum of the kinetic energies of the mechanism links and the non-inertial base, that is,

$$
U_{k}(q, v)=\frac{1}{2} v^{T} \underbrace{\left(\left[\begin{array}{cc}
I_{b} & 0 \\
0 & 0
\end{array}\right]+\sum_{i=1}^{n} M_{i}(q)\right)}_{\text {inertia matrix } M(q)} v
$$

with $M(q)$ the inertia matrix of the total system. Note that neither $U_{k}(q, v)$ nor $M(q)$ depend on the pose $g_{0 b}$ and hence the choice of inertial reference frame $\Psi_{0}$.

We can write (4) in block-form as follows

$$
\left[\begin{array}{cc}
M_{V V} & M_{q V}^{T} \\
M_{q V} & M_{q q}
\end{array}\right]\left[\begin{array}{c}
\dot{V}_{0 b}^{b} \\
\ddot{q}
\end{array}\right]+\left[\begin{array}{cc}
\bar{C}_{V V} & \bar{C}_{V q} \\
\bar{C}_{q V} & \bar{C}_{q q}
\end{array}\right]\left[\begin{array}{c}
V_{0 b}^{b} \\
\dot{q}
\end{array}\right]=\left[\begin{array}{c}
F_{b}^{b} \\
\tau
\end{array}\right]
$$

with $F_{b}^{b}$ the external wrench on the base link, expressed in coordinates $\Psi_{b}$ (such that it is collocated with the twist $\left.V_{0 b}^{b}\right)$. To compute the matrix $\bar{C}(Q, v)$ for our system, we can use the observations that $M(q)$ is independent of $g_{0 b}$, that $S(Q, \phi)$ is independent of $q$, and that $S(Q, 0) \equiv I$. Furthermore, the partial derivative of $M$ with respect to $\phi_{b}$ is zero since $M$ is independent of $g_{0 b}$, and the second term of (5) is only non-zero for the $\bar{C}_{V V}$ block of $\bar{C}(Q, v)$.
The precise computational details of the partial derivatives follow the same steps as in the classical approach [14]. To compute the partial derivatives of the adjoint matrices, one can use a relatively simple relation. If we express the velocity of joint $k$ as $V_{(k-1) k}^{(k-1)}=X_{k} \dot{q}_{k}$ for constant $X_{k}$, then the following holds:

$\frac{\partial \operatorname{Ad}_{g_{i j}}}{\partial q_{k}}=\left\{\begin{aligned} \operatorname{Ad}_{g_{i(k-1)}} \operatorname{ad}_{X_{k}} \operatorname{Ad}_{g_{(k-1) j}} & \text { for } i<k \leq j \\ -\operatorname{Ad}_{g_{i(k-1)}} \operatorname{ad}_{X_{k}} \operatorname{Ad}_{g_{(k-1) j}} & \text { for } j<k \leq i \\ 0 & \text { otherwise }\end{aligned}\right.$

To prove this, we start by writing out the spatial velocity of frame $\Psi_{k}$ with respect to $\Psi_{(k-1)}$ when $i<k \leq j$ :

$$
\hat{X}_{k} \dot{q}_{k}=\hat{V}_{(k-1) k}^{(k-1)}=\dot{g}_{(k-1) k} g_{(k-1) k}^{-1}=\frac{\partial g_{(k-1) k}}{\partial q_{k}} g_{k(k-1)} \dot{q}_{k}
$$
where $\hat{X}:=\left[\begin{array}{cc}\hat{X}_{\omega} & X_{v} \\ 0 & 0\end{array}\right]$. If we compare the first and the last
terms, we get

$$
\begin{aligned}
\frac{\partial R_{(k-1) k}}{\partial q_{k}} & =\hat{X}_{\omega} R_{(k-1) k} \\
\frac{\partial p_{(k-1) k}}{\partial q_{k}} & =\hat{X}_{\omega} p_{(k-1) k}+X_{v} .
\end{aligned}
$$

We can use this relation in the expression for the partial derivative of $\operatorname{Ad}_{g_{(k-1) k}}$ :

$$
\begin{aligned}
\frac{\partial \operatorname{Ad}_{g_{(k-1) k}}}{\partial q} & =\left[\begin{array}{cc}
\frac{\partial R_{(k-1) k}}{\partial q_{k}} & \frac{\hat{p}_{(k-1) k}}{\partial q_{k}} R_{(k-1) k}+\hat{p}_{(k-1) k} \frac{\partial R_{(k-1) k}}{\partial q_{k}} \\
0 & \frac{\partial R_{(k-1) k}}{\partial q_{k}}
\end{array}\right] \\
& =\left[\begin{array}{cc}
\hat{X}_{\omega} & \hat{X}_{v} \\
0 & \hat{X}_{\omega}
\end{array}\right]\left[\begin{array}{cc}
R_{(k-1) k} & \hat{p}_{(k-1) k} R_{(k-1) k} \\
0 & R_{(k-1) k}
\end{array}\right] \\
& =\operatorname{ad}_{X_{k}} \operatorname{Ad}_{g_{(k-1) k}}
\end{aligned}
$$

It is now straight forward to show that

$$
\begin{aligned}
\frac{\partial \operatorname{Ad}_{g_{i j}}}{\partial q_{k}} & =\operatorname{Ad}_{g_{i(k-1)}} \frac{\partial \operatorname{Ad}_{g_{(k-1) k}}}{\partial q_{k}} \operatorname{Ad}_{g_{k j}} \\
& =\operatorname{Ad}_{g_{i(k-1)}} \operatorname{ad}_{X_{k}} \operatorname{Ad}_{g_{(k-1) k}} \operatorname{Ad}_{g_{k j}} \\
& =\operatorname{Ad}_{g_{i(k-1)}} \operatorname{ad}_{X_{k}} \operatorname{Ad}_{g_{(k-1) j}} .
\end{aligned}
$$

Similarly when $j<k \leq i$.

\section{Manipulator dynamics on a forced non-inertial base}

We now simplify and specialize the dynamics equations by assuming that the motion of the platform is fully determined by external forces that are neither known nor of interest. We only assume that the relative pose $g_{0 b}$, velocity $V_{0 b}^{b}$, and acceleration $\dot{V}_{0 b}^{b}$ of the base relative to the inertial world are known from measurements. This implicitly implies that the torques applied to the internal robot joints do not influence the motion of the platform, which is a reasonable assumption in our application of a relatively small robot attached to a large moving base.

Since we are not interested in the external forces on the mechanism, we can consider just the second block-row of (11), which expresses the robot accelerations $\ddot{q}$ as a function 
of the joint torques $\tau$ as well as the non-inertial motion of the base. This can be rewritten as

$$
M_{q q} \ddot{q}+\bar{C}_{q q} \dot{q}+\underbrace{M_{q V} \dot{V}_{0 b}^{b}+\bar{C}_{q V} V_{0 b}^{b}}_{\text {inertial forces }}=\tau
$$

which partially separates the usual robot dynamics (first two terms) from the inertial forces (third and fourth term), although the matrix $\bar{C}_{q q}$ generally still depends on $V_{0 b}^{b}$. For a static base frame $\left(V_{0 b}^{b} \equiv 0\right)$, the equations reduce to the regular dynamics of an $n$-link robotic mechanism. Note that for constant $V_{0 b}^{b}$, the terms due to the non-inertial base motion generally do not drop out, since a constant twist can also contain (non-inertial) angular components. Note also that neither the inertia of the base nor the second term in (5) appear in these equations.

The terms $\bar{C}_{q V}$ and $\bar{C}_{q q}$ can be written more explicitly as

$$
\begin{aligned}
\bar{C}_{q V} & =\sum_{k=1}^{n} \frac{\partial M_{q V}}{\partial q^{k}} \dot{q}^{k}-\frac{1}{2} \frac{\partial^{T}}{\partial q}\left(\left[\begin{array}{ll}
M_{V V} & M_{q V}^{T}
\end{array}\right]\left[\begin{array}{c}
V_{0 b}^{b} \\
\dot{q}
\end{array}\right]\right) \\
\bar{C}_{q q} & =\sum_{k=1}^{n} \frac{\partial M_{q q}}{\partial q^{k}} \dot{q}^{k}-\frac{1}{2} \frac{\partial^{T}}{\partial q}\left(\left[\begin{array}{ll}
M_{q V} & M_{q q}^{T}
\end{array}\right]\left[\begin{array}{c}
V_{0 b}^{b} \\
\dot{q}
\end{array}\right]\right)
\end{aligned}
$$

This approach can be used to obtain the dynamics equations for an arbitrary $n$-link mechanism attached to a non-inertial base. Specific examples are presented in Section IV.

\section{Gravitational forces}

Finally we include the gravitational forces. Let the wrench associated with the gravitational force of link $i$ with respect to coordinate frame $\Psi_{i}$ be given by

$$
F_{g}^{i}=\left[\begin{array}{c}
f_{g} \\
\hat{r}_{g}^{i} f_{g}
\end{array}\right]=-m_{i} g\left[\begin{array}{c}
R_{0 i} e_{z} \\
\hat{r}_{g}^{i} R_{0 i} e_{z}
\end{array}\right]
$$

where $e_{z}=\left[\begin{array}{lll}0 & 0 & 1\end{array}\right]^{\top}$ and $r_{g}^{i}$ is the center of mass of link $i$ expressed in frame $\Psi_{i}$. In our case $\Psi_{i}$ is chosen so that $r_{g}^{i}$ is in the origin of $\Psi_{i}$ so we have $r_{g}^{i}=0$. The equivalent joint torque associated with link $i$ is given by

$$
\tau_{g}^{i}=J_{i}(q) \operatorname{Ad}_{g_{0 i}}^{\top}(Q) F_{g}^{i}(Q)
$$

where $J_{i}$ is the geometric Jacobian and $\operatorname{Ad}_{g_{0 i}}=\operatorname{Ad}_{g_{0 b}} \operatorname{Ad}_{g_{b i}}$ is the transformation from the inertial frame to frame $i$. We note that both $R_{0 i}$ and $\operatorname{Ad}_{g_{0 i}}$ depend on the base configuration with respect to the inertial frame. The total effect of the gravity from all the links is then given by $\tau_{g}=\sum_{i=1}^{n} \tau_{g}^{i}$ which enters Equation (16) the same way as the control torque.

\section{Compensation Using Motion Planning}

In general there are two ways to deal with the inertial forces. We can try to compensate for the effects in the controller or in the motion planning algorithm. In the first method we cancel the effects of the inertial forces in the feed-forward terms of the controller. Consider the control law

$$
\tau=\tau_{f f}+\tau_{P D}
$$

where

$$
\begin{aligned}
\tau_{f f}= & \underbrace{M_{q q} \ddot{q}_{d}+\bar{C}_{q q} \dot{q}_{d}}_{\text {tracking terms }}+\underbrace{M_{q V} \dot{V}_{0 b}^{b}+\bar{C}_{q V} V_{0 b}^{b}}_{\text {compensation for inertial forces }} \\
& -\underbrace{\sum_{n=1}^{n}\left(J_{i} \operatorname{Ad}_{g_{0 i}}^{\top} F_{g}^{i}\right)}_{\text {gravity compensation }} \\
\tau_{P D}= & \underbrace{K_{P}\left(q_{d}-q\right)+K_{D}\left(\dot{q}_{d}-\dot{q}\right)}_{\text {PD-controller }}
\end{aligned}
$$

This is the standard augmented PD control law which in our case also compensates for the inertial forces. As we are mainly interested in the feed-forward terms, we will assume perfect tracking, i.e. $q(t)=q_{d}(t)$. With this control law the non-inertial and gravitational terms are regarded as disturbances and are canceled.

When large inertial forces are present, canceling these terms may be very energy demanding. Thus, instead of regarding these terms as disturbances, we will find the trajectory for which the non-inertial and gravitational terms coincide with the tracking terms to an as large extent as possible. In doing this, the inertial forces will contribute to the desired motion instead of working against it. This will reduce the wear and tear on the manipulator, require less actuator torques and allow more accurate manipulation.

Given the dynamic equations, the initial position $q_{0}$, and desired end position $q_{\text {des }}$ in joint coordinates, we want to find the optimal trajectory given by the minimum of the cost function $P$, i.e.

$$
P_{\min }=\min _{q(t)} \int_{t=T_{0}}^{T_{1}} P(\tau) d t
$$

where $P(\tau)$ is some cost function representing the torque required for the motion,

$$
\begin{aligned}
& q\left(T_{0}\right)=q_{0}, \\
& q\left(T_{1}\right)=q_{\text {des }},
\end{aligned}
$$

are the vectors describing the initial and end positions of all the joints and

$$
M_{q q} \ddot{q}+\bar{C}_{q q} \dot{q}+M_{q V} \dot{V}_{0 b}^{b}+\bar{C}_{q V} V_{0 b}^{b}-\tau_{g}=\tau
$$

determines the dynamics of the system.

The global solution to this problem is generally very complex. Assuming $g_{0 b}, V_{0 b}^{b}(t)$ and $\dot{V}_{0 b}^{b}(t)$ known, we first need to compute $M_{q q}(q), \bar{C}_{q q}\left(q, \dot{q}, V_{0 b}^{b}\right), M_{q V}(q)$ and $\bar{C}_{q V}\left(q, \dot{q}, V_{0 b}^{b}\right)$. Then we need to find the optimal trajectory $(q(t), \dot{q}(t), \ddot{q}(t))$ which requires the least amount of torque. Both these operations are computationally very demanding.

\section{EXAMPLES}

We now present specific examples of how the previous modeling and planning methods can be applied in case of specific robot motion objectives and given base motion. Here, we make specific choices as to how to discretize and approximate the problem to make it solvable; future work will investigate different and more general approaches. 


\section{A. Parameterization of joint motion}

To reduce the search space, we assume that the shape of each joint trajectory is given so that we only need to find the starting time and the length of the motion for each joint. We also consider a cost function $P(\tau)$ that is quadratic in $\tau$ and thus reduce the problem to

$$
P_{\text {min }}=\min _{t_{0}, t_{1}} \int_{t=T_{0}}^{T_{1}} \tau^{\top} D \tau d t
$$

where $D$ is a positive definite matrix that defines a metric in $\tau$-space, $t_{0}=\left[\begin{array}{lllll}t_{1,0} & \cdots & t_{i, 0} & \cdots & t_{n, 0}\end{array}\right]^{\top}$ are the starting times and $t_{1}=\left[\begin{array}{lllll}t_{1,1} & \cdots & t_{i, 1} & \cdots & t_{n, 1}\end{array}\right]^{\top}$ are the end times for the $n$ joints, which can all be chosen independently, with the restriction that $T_{0} \leq t_{i, 0}<t_{i, 1} \leq T_{1}$ for all $i$ and for a fixed prescribed time interval $\left(T_{0}, T_{1}\right)$. We choose sinusoidal joint motions given by

$$
\begin{aligned}
& \ddot{q}_{i}(t)=a_{i} \sin \left(b_{i}\left(t-t_{i, 0}\right)\right), \\
& \dot{q}_{i}(t)=\frac{a_{i}}{b_{i}}-\frac{a_{i}}{b_{i}} \cos \left(b_{i}\left(t-t_{i, 0}\right)\right), \\
& q_{i}(t)=q_{i, 0}+\frac{a_{i}}{b_{i}}\left(t-t_{i, 0}\right)-\frac{a_{i}}{b_{i}^{2}} \sin \left(b_{i}\left(t-t_{i, 0}\right)\right),
\end{aligned}
$$

for $t \in\left(t_{i, 0}, t_{i, 1}\right)$ and $q_{i}(t)$ constant otherwise. The boundary conditions $q_{i}\left(t_{i, 0}\right)=q_{i, 0}$ and $q_{i}\left(t_{i, 1}\right)=q_{i, \text { des }}$ give rise to the following two equations

$$
a_{i}=\frac{\left(q_{i, d e s}-q_{i, 0}\right) b_{i}^{2}}{2 \pi} \quad b_{i}=\frac{2 \pi}{t_{i, 1}-t_{i, 0}}
$$

and hence the motion is fully parameterized by $t_{i, 0}$ and $t_{i, 1}$ for given $q_{0}$ and $q_{d e s}$. The motion planning problem is thus reduced to finding the optimal time intervals $\left(t_{i, 0}, t_{i, 1}\right)$ for all joints $i=1, \ldots, n$.

\section{B. Base motion}

The environmental disturbances that affect the platform motion are wind, waves and ocean currents. The ocean currents are low frequency disturbances and will not affect the manipulator dynamics. It is common to assume the principle of superposition when considering wave and wind disturbances [16] and they are normally modeled in the frequency spectrum. Many good models of the ship motion for different sea states are available in literature [16], [17].

The platform motion is modeled as $g_{0 b}(t) \in S E(3)$. Large marine vessels are often found to have a characteristic motion which we can represent as a vector subspace of se(3). For the purpose of this paper, we will estimate the main angular motion of the platform somewhat roughly by a sinusoidal motion in $S O(2)$. Assume that the waves hit the platform with a velocity in the direction of the $y$-axis in $\Psi_{b}$. The platform pose and acceleration are then given by an angular motion about the $x$-axis:

$$
\phi=A \sin (B t)
$$

This is a simplified motion, but the dynamic equations are valid for any motion in $S E(3)$. Specific examples of how this base motion affects the manipulator motion are shown in the following.

\section{1-DoF manipulator}

Consider first a mechanism with a single 1-DoF prismatic joint located at $p_{b 1}=\left[\begin{array}{lll}0 & 0 & l_{1}\end{array}\right]^{\top}$ in $\Psi_{b}$ and moving in the direction of the $y$-axis. Let the base motion be given as in (28). We set $m=1$ and the dynamics equations reduce to

$$
\tau=\ddot{q}-l_{1} \ddot{\phi}-q \dot{\phi}^{2}-g \sin (\phi)
$$

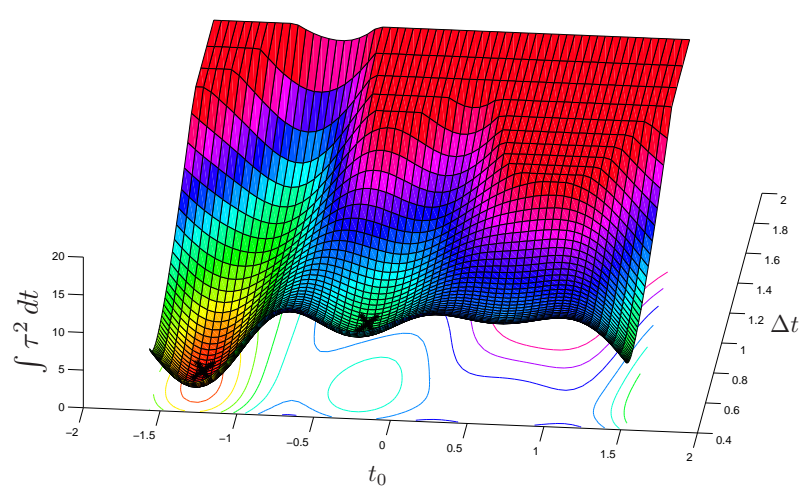

Fig. 2. The torques needed to move the prismatic joint from $q_{0}=0$ to $q_{\text {des }}=1.5$ for high frequency base motion $\phi(t)=-1 / 5 \sin (2 t)$ plotted with respect to the start time $t_{0}$ and the motion length $\Delta t=t_{1}-t_{0}$. The minima found are marked with an "X".

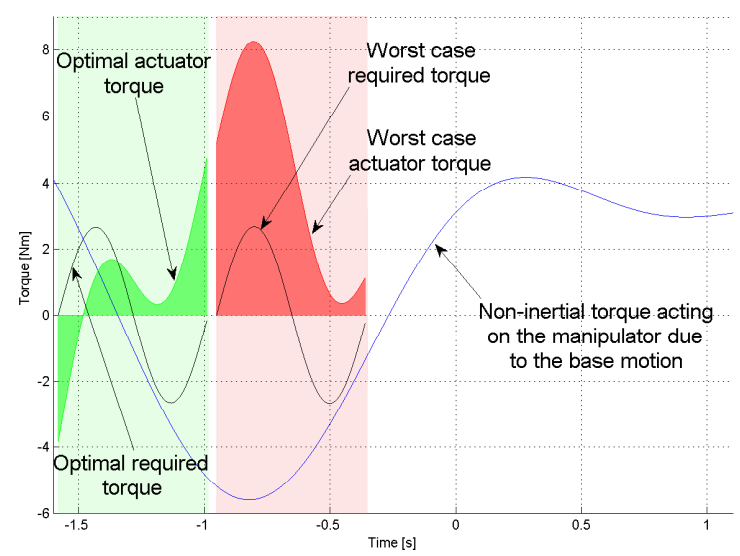

Fig. 3. Optimal and worst case trajectories. The required torque is the total torque required for the desired joint motion, which is the sum of the actuator and the inertial torques. The actuator torque is the torque applied by the actuator so that the total torque is equal to the required torque, i.e. the inertial torques subtracted from the required torque. This is the torque to be minimized. The optimal interval is found at $t=[-1.58-0.98]$. The worst case is found at $t=[-0.95-0.35]$. The integrated torque (squared) for the optimal solution is 0.87 and 11.38 for the worst case. As the length of the motion increases ( $\Delta t$ increases in Figure 2), the integrated torque increases unbounded, thus the worst case starting point search is performed with a fixed motion length of $0.6 \mathrm{~s}$.

We start by approximating the base motion given in (28) by the Taylor approximation

$$
\sin (x) \approx x-\frac{x^{3}}{3 !}+\frac{x^{5}}{5 !}-\frac{x^{7}}{7 !}+\mathcal{O}\left(x^{9}\right) .
$$


We can write $\phi(t), \dot{\phi}(t)$ and $\ddot{\phi}(t)$ as

$$
\begin{aligned}
& \phi(t) \approx A\left(B t-\frac{(B t)^{3}}{3 !}+\frac{(B t)^{5}}{5 !}-\frac{(B t)^{7}}{7 !}+\mathcal{O}\left(t^{9}\right)\right), \\
& \dot{\phi}(t) \approx A B\left(1-\frac{(B t)^{2}}{2 !}+\frac{(B t)^{4}}{4 !}-\frac{(B t)^{6}}{6 !}+\mathcal{O}\left(t^{8}\right)\right), \\
& \ddot{\phi}(t) \approx A B^{2}\left(-\frac{(B t)}{1 !}+\frac{(B t)^{3}}{3 !}-\frac{(B t)^{5}}{5 !}+\mathcal{O}\left(t^{7}\right)\right) .
\end{aligned}
$$

This is typically a good approximation for one period of sinusoidal motion. We approximate the desired joint motion given by (26) in the same way.

As we have only one joint we set $t_{0}=T_{0}$ and $t_{1}=T_{1}$. The minimization problem is then reduced to

$$
P_{\text {min }}=\min _{t_{0}, t_{1}} \int_{t_{0}}^{t_{1}}\left(\ddot{q}-l_{1} \ddot{\phi}-q \dot{\phi}^{2}-g \sin (\phi)\right)^{2} d t
$$

which after substituting the Taylor approximations reduces to the problem of finding the minimum of a polynomial equation. We can now quickly find the optimal solution with respect to the start and end times $t_{0}$ and $t_{1}$. We define the search space as the time interval for which the Taylor approximation is accurate, i.e., $\left(t_{0}, t_{1}\right) \in\left(-t_{T}, t_{T}\right)$ where $2 t_{T}$ is the wave period of the principal frequency of the waves. Figure 2 illustrates the value of the integral (31) for different start and end times for $\phi(t)=-1 / 5 \sin (2 t), q_{0}=0$ and $q_{\text {des }}=1.5$. The optimal and worst case trajectories are shown in Figure 3.

\section{4-DoF manipulator}

The previous example can be solved efficiently as it reduces to finding the minimum of a polynomial equation. As a second example, we show how numerical methods can be used to compute optimal motion paths for the 4DoF manipulator shown in Figure 1 with realistic mass and inertia parameters. We now use the exact equations for base motion and the manipulator dynamics. The base moves along the angular motion pattern (28) at a relatively low frequency, which means the inertial forces mostly enter through a changing direction of gravity.

We solve the motion planning problem by numerically minimizing the objective function (22) and parameterize the problem as follows: each joint trajectory is given by a separate sinusoidal motion (26) with parameters $t_{i, 0}$ and $t_{i, 1}$, the total motion from start to goal is to be finished within a fixed prescribed time interval $\left(T_{0}, T_{1}\right)=(0,10)$, and the cost function is chosen as (25) with $D=10^{-6} I$ and integrated over the fixed time interval $\left(T_{0}, T_{1}\right)$. We choose the start and end configurations as

$$
\begin{aligned}
q(0) & =\left[\begin{array}{llll}
-2.5 & 0 & 0 & 0
\end{array}\right]^{\top} \\
q(T) & =\left[\begin{array}{llll}
2.5 & \frac{\pi}{2} & \frac{\pi}{2} & \frac{\pi}{2}
\end{array}\right]^{\top}
\end{aligned}
$$

and the base motion as (28) with $A=\frac{5}{4 \pi}$ and $B=\frac{2 \pi}{5}$. The motion planning problem thus amounts to finding the eight parameters (one start and end time for each joint) that minimize the total squared required torque integrated over a
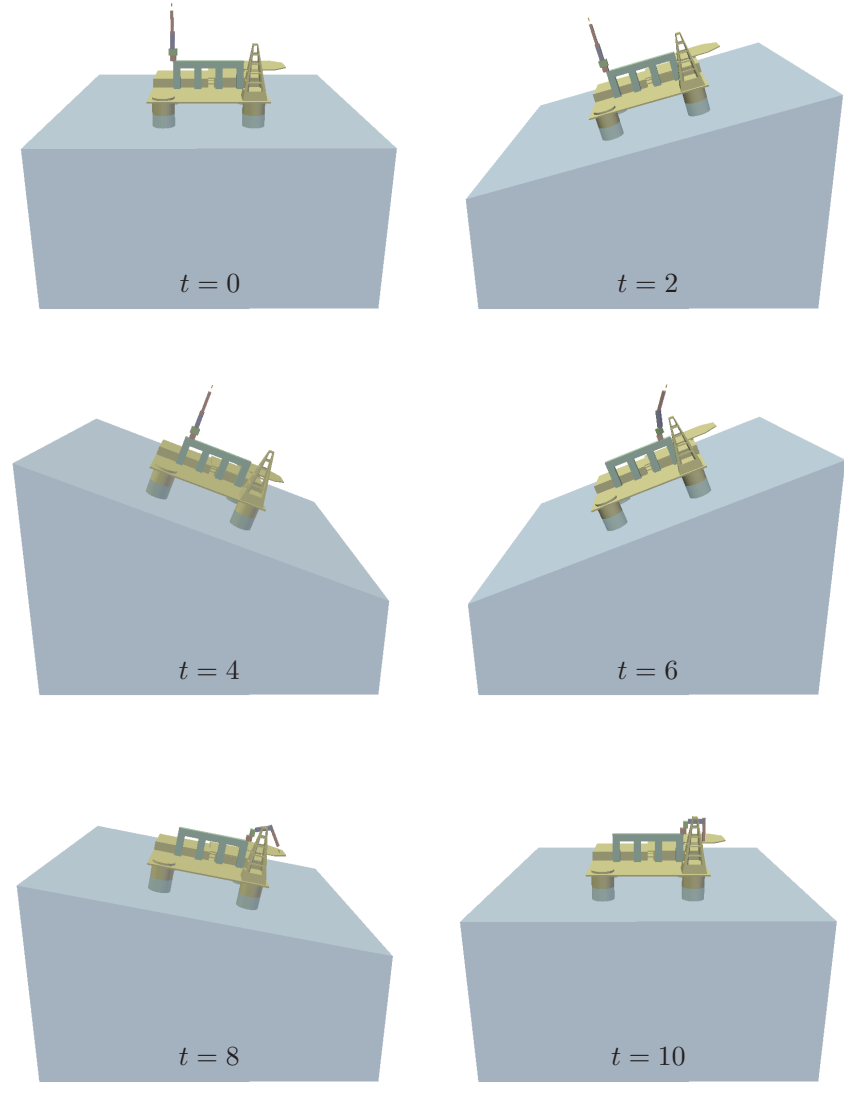

Fig. 4. Still shots from the simulation of the fully optimized trajectory corresponding to the solid lines in Figure 5.

fixed time interval while starting and finishing the robot in the required configurations.

Figures 4 and 5 show the solution obtained using Matlab's constrained minimization function fmincon. A full animation of the resulting motions can be found in the video accompaniment to this paper. Figure 4 shows still shots of the optimal solution and illustrates the sinusoidal motion of the base. Figure 5 compares three solutions: one baseline solution that simply takes the start time for each joint trajectory at $t_{i, 0}=T_{0}$ and the finish time at $t_{i, 1}=T_{1}$, one solution that optimizes the cost function assuming zero base motion (a horizontal stationary base), and one solution that optimizes the cost function taking the real base motion into account. The associated costs are 19,21, and 11, respectively.

The figure shows that, for this example, taking the base motion into account can significantly reduce the cost and hence the required torque. The joint motions optimized for a static base (dashed line) even perform worse than the baseline joint motions (dotted line) when applied during non-static base motion. When optimizing the joint motions while taking the base motion into account (solid line), the result is much improved, and the benefit of the resulting motions can be understood intuitively: the prismatic motion is delayed and shortened as to optimally use the changing gravity direction (due to base rotation) in the acceleration and deceleration 

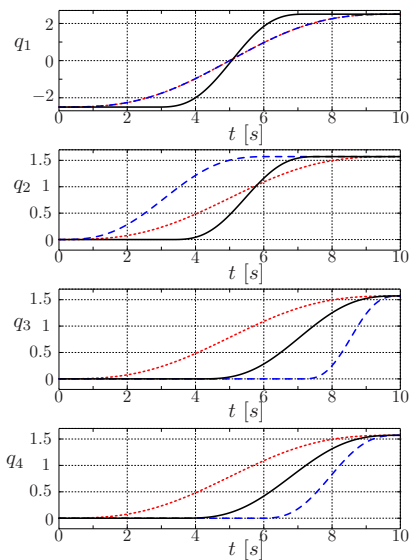

(a) Joint trajectories (m,rad).
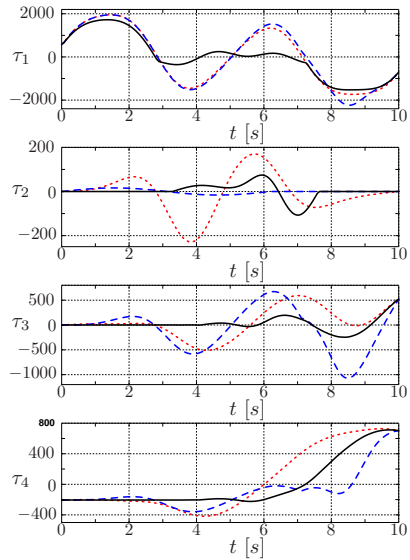

(b) Required joint torques $(\mathrm{N}, \mathrm{Nm})$

Fig. 5. Optimal motion trajectory for a 4-DoF manipulator. Three different trajectories are shown: a baseline trajectory with maximum motion duration (dotted lines), an optimized trajectory assuming zero base motion (dashed lines), and an optimized trajectory taking the correct non-zero base motion into account (solid lines).

phase, similar to the previous 1-DoF example. The resulting required actuator torque $\tau_{1}$ is thus reduced to close to zero during the motion, i.e., in the time interval $\left(t_{1,0}, t_{1,1}\right)=$ $(3.2,7.2)$. Similarly, the motion of joints 3 and 4 is delayed as to minimize the amount of time spent holding up the links against gravity.

The example shows how knowledge of the base motion can be used to significantly reduce torque requirements, even with only little freedom in the optimization (only $t_{i, 0}$ and $t_{i, 1}$ can be optimized). If the shapes of the trajectories are allowed to be changed and optimized in more detail, improvement should be even more significant. Including more parameters makes the optimization problem more complex, though, and numerical solutions may get more easily trapped in local minima.

\section{CONCLUSIONS}

The classical dynamics equations for a serial manipulator have been extended to also include the motion of a forced non-inertial base. The dynamics equations are derived using a generalized Lagrangian method. This allows us to model the base motion as a "free motion" joint serially connected with the 1-DoF joints of the manipulator.

Examples for a 1-DoF and 4-DoF manipulator mounted on a platform are presented. We include the platform motion in the dynamics and find the trajectory that takes the manipulator from an initial position to an end position with the least amount of torque and compare this with the optimal trajectory when the platform is assumed not to be moving. The simulations show that when the ship motion is known the amount of torque needed for a given task can be substantially reduced if the inertial forces are taken into account.

A possible extension for future work is to optimize the shape of the joint trajectories with more variables. Adding more details to the joint trajectories should increase performance even more. If a sufficiently accurate model of the platform can be obtained, this may allow us to compensate for the inertial forces in high accuracy applications. Another interesting topic for future work is to look into how model predictive control can be used to compensate for the platform motion.

\section{ACKNOWLEDGMENTS}

The first and third authors wish to acknowledge the support of the Norwegian Research Council and the TAIL IO project for their continued funding and support for this research. The TAIL IO project is an international cooperative research project led by StatoilHydro and an R\&D consortium consisting of ABB, IBM, Aker Kvaerner and SKF. During the work with this paper the first author was with the University of California at Berkeley. The second author is sponsored through a Rubicon grant from the Netherlands Organization for Scientific Research (NWO).

\section{REFERENCES}

[1] L. J. Love, J. F. Jansen, and F. G. Pin, "On the modeling of robots operating on ships," IEEE International Conference on Robotics and Automation, vol. 3, pp. 2436-2443, 2004.

[2] J. Kitarovic, V. Tomas, and D. Čišic, "The electronic and informatics age - a new stage in developing highly effective ships," 47th International ELMAR Symposium, pp. 385-388, 2005.

[3] S.-R. Oh, K. Mankala, S. Agrawal, and J. Albus, "Dynamic modeling and robust controller design of a two-stage parallel cable robot," IEEE International Conference on Robotics and Automation, vol. 4, pp. 3678-3683, 2004.

[4] D. C. Salzmann, "Ampelmann prototype - developing a motion compensating platform for offshore access," European Wind Energy Conference, 2007.

[5] G. Lebans, K. Wilkie, R. Dubay, D. Crabtree, and T. Edmonds, "Telerobotic shipboard handling system," OCEANS, vol. 2, pp. 12371241, 1997.

[6] K. Kosuge, M. Okuda, and T. Fukuda, "Motion control of manipulator/vehicle system floating on water," Proc. of 2nd IEEE Int'l Workshop on Advanced Motion Control, pp. 1261-1268, 1992.

[7] H. Kajita and K. Kosuge, "Force control of robot floating on the water utilizing vehicle restoring force," IEEE/RSJ International Conference on Intelligent Robot and Systems, vol. 1, pp. 162-167, 1997.

[8] T. Yoshikawa, K. Harada, and A. Matsumoto, "Hybrid position/force control of flexible-macro/rigid-micro manipulator systems," IEEE Transactions on Robotics and Automation, vol. 12, no. 4, pp. 633640, 1996.

[9] A. Bowling and O. Khatib, "Design of macro/mini manipulators for optimal dynamic performance," Proc. IEEE International Conference on Robotics and Automation,, vol. 1, pp. 449-454, 1997.

[10] S. McMillan, D. E. Orin, and R. B. McGhee, "Efficient dynamic simulation of an underwater vehicle with a robotic manipulator," IEEE Transactions on systems, man and cybernetics, vol. 25, no. 8, pp. 1194-1206, 1995.

[11] O. Egeland and J. R. Sagli, "Coordination of motion in a spacecraft/manipulator system," International Journal of Robotics Research, vol. 12 no. 4, pp. 366-379, 1993.

[12] V. Duindam and S. Stramigioli, "Lagrangian dynamics of open multibody systems with generalized holonomic and nonholonomic joints," in Proceedings of the IEEE/RSJ International Conference on Intelligent Robots and Systems, October 2007, pp. 3342-3347.

[13] _ _ "Singularity-free dynamic equations of open-chain mechanisms with general holonomic and nonholonomic joints," IEEE Transactions on Robotics, vol. 24, no. 3, pp. 517-526, June 2008.

[14] R. M. Murray, Z. Li, and S. S. Sastry, A Mathematical Introduction to Robotic Manipulation. CRC Press, 1994.

[15] V. Duindam, "Port-based modeling and control for efficient bipedal walking robots," Ph.D. dissertation, University of Twente, March 2006. [Online]. Available: http://purl.org/utwente/50829

[16] T. I. Fossen, Marine Control Systems, 3rd printing. Marine Cybernetics, 2002.

[17] N. Salvesen, E. O. Tuck, and O. M. Faltinsen, "Ship motions and sea loads," Trans SNAME, vol. 78, p. 250287, 1970. 